\title{
Neutrino Physics at the Turn of the Millenium
}

\author{
José W. F. Valle \\ Instituto de Física Corpuscular - C.S.I.C. \\ Departamento de Física Teòrica - Univ. de València, \\ Edificio Institutos de Paterna, Apartado de Correos 2085, 46071, València \\ http://neutrinos.uv.es
}

\begin{abstract}
I discuss the implications of the latest data on solar and atmospheric neutrinos which strongly indicate the need for physics beyond the Standard Model. I review the theoretical options for reconciling these data in terms of three-neutrino oscillations. Even though not implied by the data, bi-maximal models of neutrino mixing emerge as an attractive possibility. Supersymmetry with broken R-parity provides a predictive way to incorporate it, opening the possibility of testing neutrino anomalies at high-energy collider experiments such as the LHC or at the upcoming long-baseline or neutrino factory experiments. Reconciling, in addition, the hint provided by the LSND experiment requires a fourth, light sterile neutrino. The simplest theoretical scenarios are the most symmetric ones, in which two of the four neutrinos are maximally-mixed and lie at the LSND scale, while the others are at the solar mass scale. The lightness of the sterile neutrino, the nearly maximal atmospheric neutrino mixing, and the generation of $\Delta m_{\odot}^{2} \& \Delta m_{\text {atm }}^{2}$ all follow naturally from the assumed lepton-number symmetry and its breaking. These two basic schemes can be distinguished at neutral-current-sensitive solar \& atmospheric neutrino experiments such as the Sudbury Neutrino Observatory. However, underground experiments have not yet proven neutrino masses, since there is a variety of alternative mechanisms. For example, flavour changing interactions can play an important rôle in the explanation of solar and of contained atmospheric data and could be tested through effects such as $\mu \rightarrow e+\gamma, \mu-e$ conversion in nuclei, unaccompanied by neutrino-less double beta decay. Conversely, the room is still open for heavy unstable neutrinos. A short-lived $\nu_{\mu}$ might play a rôle in the explanation of the atmospheric data. Finally, in the presence of a sterile neutrino $\nu_{s}$, a long-lived $\nu_{\tau}$ in the $\mathrm{MeV}$ range could delay the time at which the matter and radiation contributions to the energy density of the Universe become equal, reducing the density fluctuations on the smaller scales, and rescuing the standard cold dark matter scenario for structure formation. In this case the light $\nu_{e}, \nu_{\mu}$ and $\nu_{s}$ would account for the solar \& atmospheric data.
\end{abstract}

\section{Introduction}

Undoubtedly the solar [1, 2, 3, 国 and atmospheric [5, 6] neutrino problems provide the two most important milestones in the search for physics beyond the Standard Model (SM). Of particular importance has been the recent confirmation by the Super-Kamiokande collaboration [6] of the zenith-angle-dependent deficit of atmospheric neutrinos. Altogether solar and atmospheric data give a strong evidence for $\nu_{e}$ and $\nu_{\mu}$ conversions, respectively. Neutrino conversions are a 
natural consequence of theories beyond the Standard Model [7]. The first example is oscillations of small-mass neutrinos. The simplest way to account for the lightness of neutrinos is in the context of Majorana neutrinos: their mass violates lepton number. Its most obvious consequences would be processes such as neutrino-less double beta decay [8], or CP violation properties of neutrinos [9], so far unobserved. Neutrino masses could be hierarchical, with the light $\nu_{\tau}$ much heavier than the $\nu_{\mu}$ and $\nu_{e}$. While solar neutrino rates favour the small mixing angle (SMA) MSW solution, present data on the recoil-electron spectrum prefer the large mixing MSW [10] (LMA) solution [11]. When interpreted in terms of neutrino oscillations, the observed atmospheric neutrino zenith-angle-dependent deficit clearly indicates that the mixing involved is maximal. In short we have the intriguing possibility that, unlike the case of quarks, neutrino mixing is bi-maximal. Supersymmetry with broken $\mathrm{R}$-parity provides an attractive origin for bi-maximal neutrino oscillations, which can be tested not only at the upcoming longbaseline or neutrino factory experiments but also at high-energy collider experiments such as the LHC.

One should however bear in mind that there is a variety of alternative solutions to the neutrino anomalies. Just as an example let me stress the case for lepton flavour violating neutrino transitions, which can arise without neutrino masses 12, 13, 14. They may still fit present solar [15] and contained atmospheric [16] data pretty well. They may arise in models with extra heavy leptons 117, 18, 19, 20] and in supergravity theories [21]. A possible signature of theories leading to FC interactions would be the existence of sizeable flavour non-conservation effects, such as $\mu \rightarrow e+\gamma, \mu-e$ conversion in nuclei, unaccompanied by neutrino-less double beta decay if neutrinos are massless. In contrast to the intimate relationship between the latter and the non-zero Majorana mass of neutrinos due to the Black-Box theorem [8] there is no fundamental link between lepton flavour violation and neutrino mass. Other possibilities involve neutrino decays [22] and transition magnetic moments [23] coupled to either to regular [24, 25] or to random magnetic fields [26].

In addition to the solar and atmospheric neutrino data from underground experiments there is also some indication for neutrino oscillations from the LSND experiment [27, 28]. Barring exotic neutrino conversion mechanisms one requires three mass scales in order to reconcile all of these hints, hence the need for a light sterile neutrino [29, 30, 31]. Out of the four neutrinos, two of them lie at the solar neutrino scale and the other two maximally-mixed neutrinos are at the HDM/LSND scale. The prototype models proposed in [29, 30] enlarge the $S U(2) \otimes U(1)$ Higgs sector in such a way that neutrinos acquire mass radiatively, without unification nor seesaw. The LSND scale arises at one-loop, while the solar and atmospheric scales come in at the two-loop level, thus accounting for the hierarchy. The lightness of the sterile neutrino, the nearly maximal atmospheric neutrino mixing, and the generation of the solar and atmospheric neutrino scales all result naturally from the assumed lepton-number symmetry and its breaking. Either $\nu_{e}-\nu_{\tau}$ conversions explain the solar data with $\nu_{\mu}-\nu_{s}$ oscillations accounting for the atmospheric deficit [29], or else the rôles of $\nu_{\tau}$ and $\nu_{s}$ are reversed [30]. These two basic schemes have distinct implications at future solar \& atmospheric neutrino experiments with 


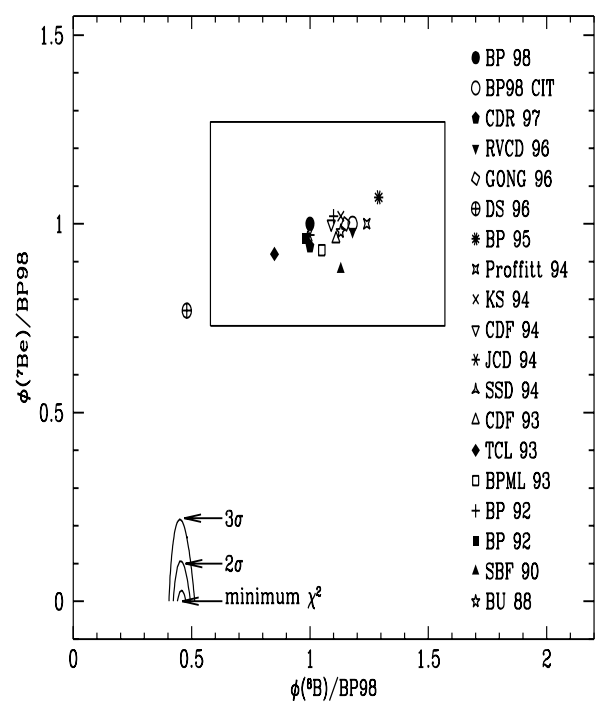

Figure 1: SSM predictions, from ref. 33.

good sensitivity to neutral current neutrino interactions. Cosmology can also place restrictions on these four-neutrino schemes 32.

\section{Indications for New Physics}

The most solid hints in favour of new physics in the neutrino sector come from underground experiments on solar [1, 2, 3, 4] and atmospheric [5, 6] neutrinos. The most recent data correspond to 825-day solar [4] and 52 kton-yr atmospheric data samples, respectively [6].

\section{$2.1 \quad$ Solar Neutrinos}

The solar neutrino event rates recorded at the radiochemical Homestake, Gallex and Sage experiments are summarized as: $2.56 \pm 0.22 \mathrm{SNU}$ (chlorine), 72.3 $\pm 5.6 \mathrm{SNU}$ (Gallex and Sage) [1, 2]. Note that only the gallium experiments are sensitive to the solar $p p$ neutrinos. On the other hand the ${ }^{8} \mathrm{~B}$ flux from Super-Kamiokande water Cerenkov experiment is $(2.44 \pm$ $0.08) \times 10^{6} \mathrm{~cm}^{-2} \mathrm{~s}^{-1}$ 畂. In Fig. (1) one can see the predictions of various standard solar models in the plane defined by the ${ }^{7} \mathrm{Be}$ and ${ }^{8} \mathrm{~B}$ neutrino fluxes, normalized to the predictions of the BP98 solar model [34]. Abbreviations such as BP95, identify different solar models, as given in ref. [35]. The rectangular error box gives the $3 \sigma$ error range of the BP98 fluxes. On the other hand the values of these fluxes indicated by present data on neutrino event rates are shown by the contours in the lower-left part of the figure. The best-fit ${ }^{7} \mathrm{Be}$ neutrino flux is negative! The theoretical predictions clearly lie well away from the $3 \sigma$ contour, strongly suggesting the need for new particle physics in order to account for the data [36]. Since possible non-standard astrophysical solutions are rather constrained by helioseismology studies [33, 37] one is led to assume the existence of neutrino conversions, such as those induced by very small neutrino masses. Possibilities include the MSW effect [10], vacuum neutrino oscillations [38, 39] and, 


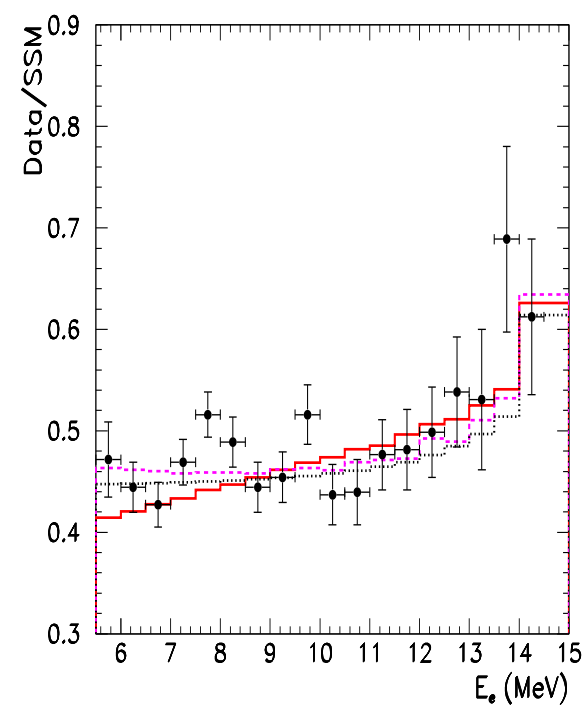

Figure 2: Expected normalized recoil electron energy spectra versus 825-day SuperKamiokande data, from [11].

possibly, flavour changing neutrino interactions [15]. Moreover, if neutrinos have transition magnetic moments then one may have, in addition, the possibility of Majorana neutrino SpinFlavour Precessions 23]. Based upon these there emerge two new solutions to the solar neutrino problem: the Resonant [24, 25] and the Aperiodic Spin-Flavour Precession mechanisms [26], based on regular and random magnetic fields, respectively.

The recent 825-day data sample [4] presents no major surprises, except that the recoil energy spectrum produced by solar neutrino interactions shows more events in the highest bins. Barring the possibly of poorly understood energy resolution effects, it has been noted 40] that if the flux for neutrinos coming from the ${ }^{3} \mathrm{He}+p \rightarrow{ }^{4} \mathrm{He}+e^{+}+\nu_{e}$, the so-called hep reaction, is well above the (uncertain) SSM predictions, then this could significantly influence the electron energy spectrum produced by solar neutrino interactions in the high recoil region, with hardly any effect at lower energies. Fig. 2 shows the expected normalized recoil electron energy spectrum compared with the most recent experimental data 4 . The solid line represents the prediction for the best-fit SMA solution with free ${ }^{8} B$ and hep normalizations (0.69 and 12 respectively), while the dotted line gives the corresponding prediction for the best-fit LMA solution (1.15 and 34 respectively). Finally, the dashed line represents the prediction for the best no-oscillation scheme with free ${ }^{8} B$ and hep normalizations ( 0.44 and 14 , respectively). Clearly the spectra with enhanced hep neutrinos provide better fits to the data. However Fiorentini et al [41] have argued that the required hep amount is too large to accept on theoretical grounds. We look forward to the improvement of the situation. The increasing rôle played rate-independent observables such as the spectrum, as well as seasonal and day-night asymmetries will eventually select amongst different solutions of the solar neutrino problem.

The required solar neutrino parameters are determined through a $\chi^{2}$ fit of the experimental data. In Fig. (3) we show the allowed regions in $\Delta m^{2}$ and $\sin ^{2} \theta$ from the measurements of 


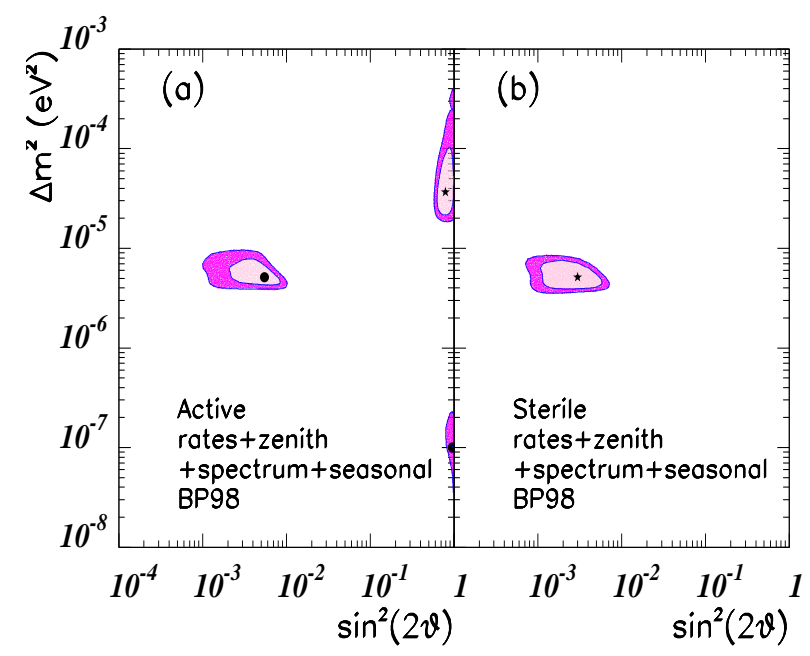

Figure 3: Solar neutrino parameters at $90 \%$ and $99 \%$ CL for 2-flavour MSW neutrino conversions with 825-day SK data sample in the BP98 model, from 11.

the total event rates at the Chlorine, Gallium and Super-Kamiokande (825-day data sample) experiments, combined with the zenith angle distribution, the recoil energy spectrum and the seasonal dependence of the event rates, observed in Super-Kamiokande. Panels (a) and (b) correspond to active-active and active-sterile oscillations, respectively. The best-fit points in each case are indicated by a star [11], while the local best-fit points are indicated by a dot. An analysis with free ${ }^{8} B$ and hep normalizations has also been given in [11] and does not change significantly the allowed regions.

One notices from the analysis that rate-independent observables, such as the electron recoil energy spectrum and the day-night asymmetry (zenith angle distribution), are playing an increasing rôle in the determination of solar neutrino parameters [11]. An observable which has been neglected in most analyses of the MSW effect and which could be sizeable in the large mixing angle regions (LMA and LOW) is the seasonal dependence in the solar neutrino flux which would result from the regeneration effect at the Earth and which has been discussed in ref. [42]. This should play a more significant rôle in future investigations.

A theoretical issue which has raised some interest recently is the study of the possible effect of random fluctuations in the solar matter density [43, 44, 45]. The possible existence of such noise fluctuations at a few percent level is not excluded by present helioseismology studies. In Fig. (4) we show averaged solar neutrino survival probability as a function of $E / \Delta m^{2}$, for $\sin ^{2} 2 \theta=0.01$. This figure was obtained via a numerical integration of the MSW evolution equation in the presence of noise, using the density profile in the Sun from BP95 in ref. [35], and assuming that the correlation length $L_{0}$ (which corresponds to the scale of the fluctuation) is $L_{0}=0.1 \lambda_{m}$, where $\lambda_{m}$ is the neutrino oscillation length in matter. An important assumption in the analysis is that $l_{\text {free }} \ll L_{0} \ll \lambda_{m}$, where $l_{\text {free }} \sim 10 \mathrm{~cm}$ is the mean free path of the electrons in the solar medium. The fluctuations may strongly affect the ${ }^{7}$ Be neutrino 


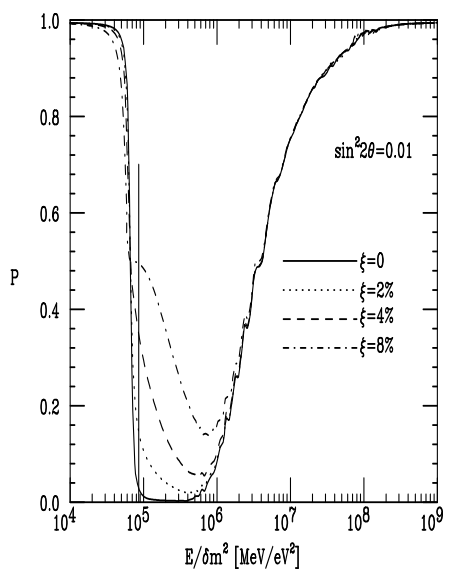

Figure 4: Solar neutrino survival probability in a noisy Sun, from ref. [44]

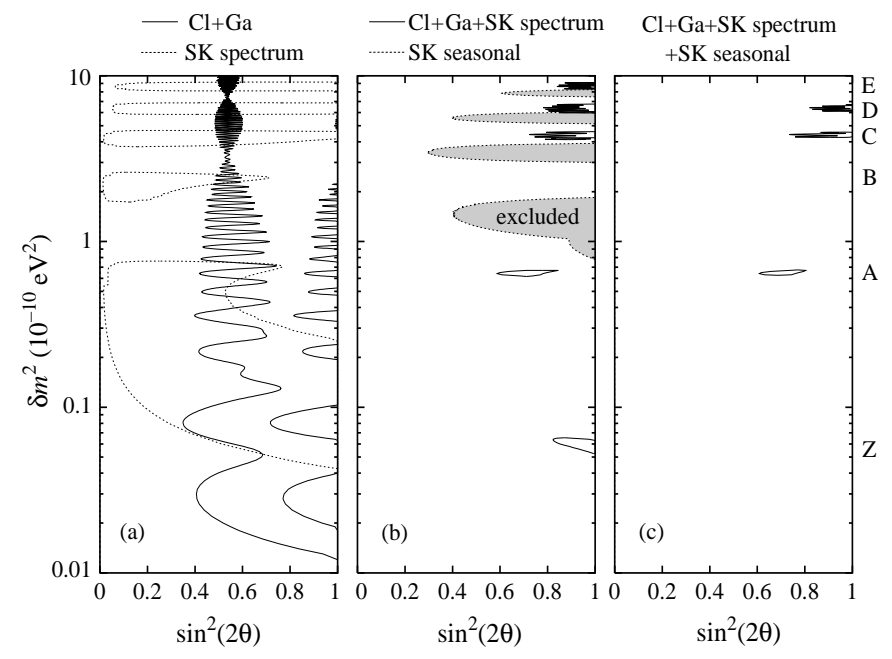

Figure 5: Vacuum oscillation parameters, from ref. 47]

component of the solar neutrino spectrum so that the Borexino experiment should provide an ideal test, if sufficiently small errors can be achieved. The potential of Borexino in probing the level of solar matter density fluctuations provides an additional motivation for the experiment 46.

The most popular alternative solution to the solar neutrino problem is the vacuum oscillation solution [38] which clearly requires large neutrino mixing and the adjustment of the oscillation length so as to coincide roughly with the Earth-Sun distance. Fig. 5 shows the regions of just-so oscillation parameters at the $95 \%$ CL obtained in a recent fit of the data, including both the rates, the recoil energy spectrum and seasonal effects, which are expected in this scenario [48] and could potentially help in discriminating it from the MSW scenario.

\subsection{Atmospheric Neutrinos}

Neutrinos produced as decay products in hadronic showers from cosmic ray collisions with nuclei in the upper atmosphere have been observed in several experiments [49, 50, 51, 52, 53, 54, 55]. 
There has been a long-standing discrepancy between the predicted and measured $\mu / e$ ratio of the muon $\left(\nu_{\mu}+\bar{\nu}_{\mu}\right)$ over the electron atmospheric neutrino flux $\left(\nu_{e}+\bar{\nu}_{e}\right)$ [56. The anomaly has been found both in water Cerenkov experiments (Kamiokande, Super-Kamiokande and IMB) as well as in the iron calorimeter Soudan2 experiment. Negative experiments, such as Frejus and Nusex have much larger errors. Although individual $\nu_{\mu}$ or $\nu_{e}$ fluxes are only known to within $30 \%$ accuracy, their ratio is predicted to within $5 \%$ over energies varying from $0.1 \mathrm{GeV}$ to $100 \mathrm{GeV}$ [57]. The most important feature of the atmospheric neutrino data sample [6] is that it exhibits a zenith-angle-dependent deficit of muon neutrinos. Experimental biases and uncertainties in the prediction of neutrino fluxes and cross sections are unable to explain the data.

The most popular way to account for this anomaly is in terms of neutrino oscillations. It has already been noted [58] that the Chooz reactor data [59] excludes the $\nu_{\mu} \rightarrow \nu_{e}$ channel, when all experiments are combined. So I concentrate here on the other possible oscillation channels.

The results of the most recent $\chi^{2}$ fit of the Super-Kamiokande atmospheric neutrino data in the framework of the neutrino oscillation hypothesis can be seen in Fig. (6), taken from ref. [60]. This analysis updates previous studies in ref. [58 and [61 and includes the upgoing muon event samples. This figure shows the allowed regions of oscillation parameters at 90 and $99 \%$ CL. Notice that matter effects lead to differences between the allowed regions for the various channels. For $\nu_{\mu} \rightarrow \nu_{s}$ with $\Delta m^{2}>0$ matter effects enhance the oscillations for neutrinos and therefore smaller values of the vacuum mixing angle would lead to larger conversion probabilities, so that the regions are larger than compared to the $\nu_{\mu} \rightarrow \nu_{\tau}$ case. For $\nu_{\mu} \rightarrow \nu_{s}$ with $\Delta m^{2}<0$ the matter enhancement occurs only for anti-neutrinos, suppressing the conversion in $\nu_{\mu}$ 's. Since the yield of atmospheric neutrinos is bigger than that of antineutrinos, clearly the matter effect suppresses the overall conversion probability. Therefore one needs in this case a larger value of the vacuum mixing angle. This trend can indeed be seen by comparing the regions in different columns of Fig. (6).

Notice that in all channels where matter effects play a rôle the range of acceptable $\Delta m^{2}$ is slightly shifted towards larger values, as compared with the $\nu_{\mu} \rightarrow \nu_{\tau}$ case. This follows from the relation between mixing in vacuo and in matter. In fact, away from the resonance region, independently of the sign of the matter potential, there is a suppression of the mixing inside the Earth. As a result, the lower allowed $\Delta m^{2}$ value is higher than for the $\nu_{\mu} \rightarrow \nu_{\tau}$ channel.

Concerning the quality of the fits we note that the best-fit to the full sample is obtained for the $\nu_{\mu} \rightarrow \nu_{\tau}$ channel, although from the global analysis oscillations into sterile neutrinos cannot be ruled out. There is also an improvement in the quality of the fits to the contained events as compared to previous analysis performed with lower statistics [58]. These features can be easily understood by looking at the predicted zenith angle distribution of the different event types for the various oscillation channels shown in Fig. (7) and Fig. (8). From Fig. (7) one can see the excellent agreement between the observed distributions of e-like events and the SM predictions. This has led to an improvement of the quality of the fit for any conversion mechanism that 


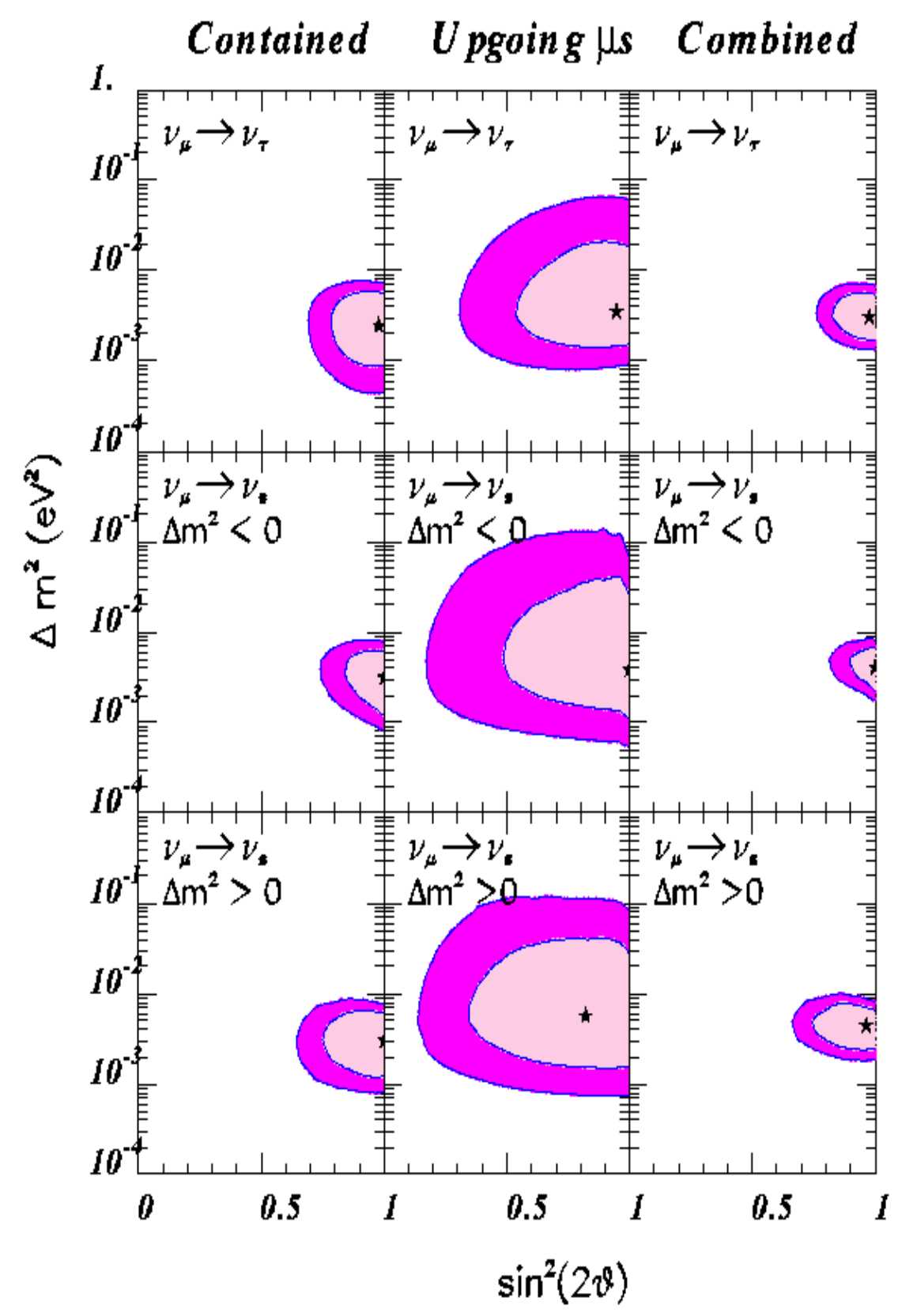

Figure 6: Allowed regions of the oscillation parameters for various Super-Kamiokande data samples and oscillation channels, as labelled in the figure. Best-fit points are denoted by a star in each case. 


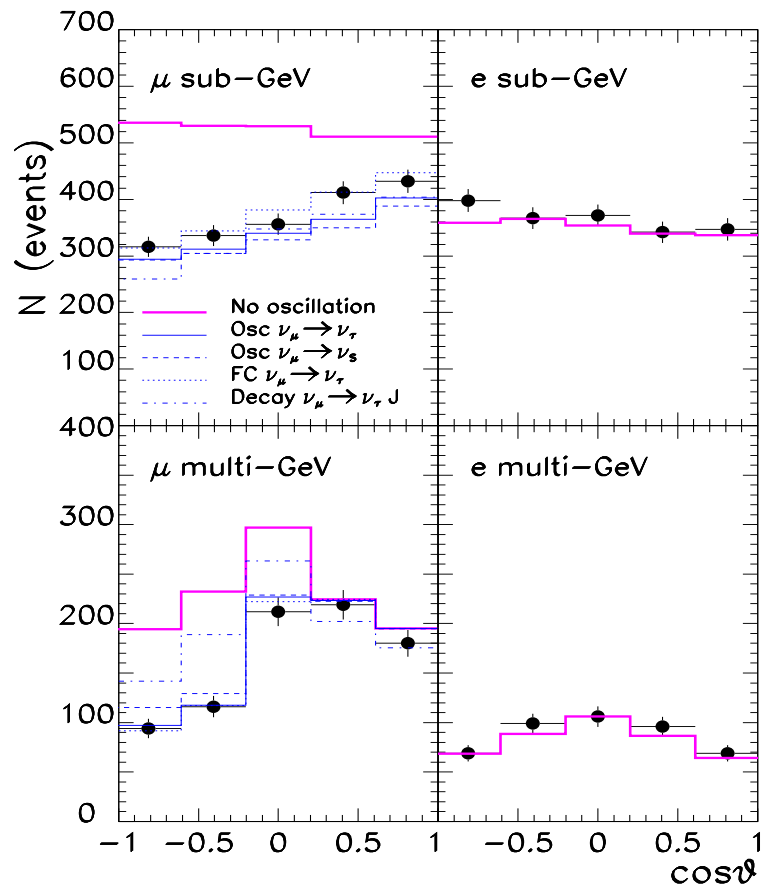

Figure 7: Angular distributions for contained Super-Kamiokande events, together with the SM prediction (no-oscillation) and the predictions for the best-fit points to the contained event data in various conversion mechanisms labelled in the figure. The error in the experimental points is only statistical.

only involves muons. From Fig. (8) one can also see that due to matter effects the distributions for upgoing muons in the case of $\nu_{\mu} \rightarrow \nu_{s}$ are flatter than for $\nu_{\mu} \rightarrow \nu_{\tau}$ [62]. The data show a somewhat steeper angular dependence which is better described by $\nu_{\mu} \rightarrow \nu_{\tau}$ oscillations. In order to exploit this feature the Super-Kamiokande collaboration has presented a preliminary partial analysis of the angular dependence of the through-going muon data in combination with the up-down asymmetry of partially contained events which seems indeed to disfavour $\nu_{\mu} \rightarrow \nu_{s}$ oscillations at the $2-\sigma$ level [50]. For a comparison of the oscillation parameters as determined from the atmospheric data with the sensitivity of the present accelerator and reactor experiments, as well as the expectations of upcoming long-baseline experiments see ref. [58].

\subsection{LSND}

The Los Alamos Meson Physics Facility looked for $\bar{\nu}_{\mu} \rightarrow \bar{\nu}_{e}$ oscillations using $\bar{\nu}_{\mu}$ from $\mu^{+}$ decay at rest [27]. The $\bar{\nu}_{e}$ 's are detected via the reaction $\bar{\nu}_{e} p \rightarrow e^{+} n$, correlated with a $\gamma$ from $n p \rightarrow d \gamma(2.2 \mathrm{MeV})$. The results indicate $\bar{\nu}_{\mu} \rightarrow \bar{\nu}_{e}$ oscillations, with an oscillation probability of $\left(0.31_{-0.10}^{+0.11} \pm 0.05\right) \%$, leading to the oscillation parameters shown in Fig. (9). The shaded regions are the favoured likelihood regions given in ref. [27]. The curves show the $90 \%$ and $99 \%$ likelihood allowed ranges from LSND, and the limits from BNL776, KARMEN1, Bugey, CCFR, and NOMAD. A search for $\nu_{\mu} \rightarrow \nu_{e}$ oscillations has also been conducted by 


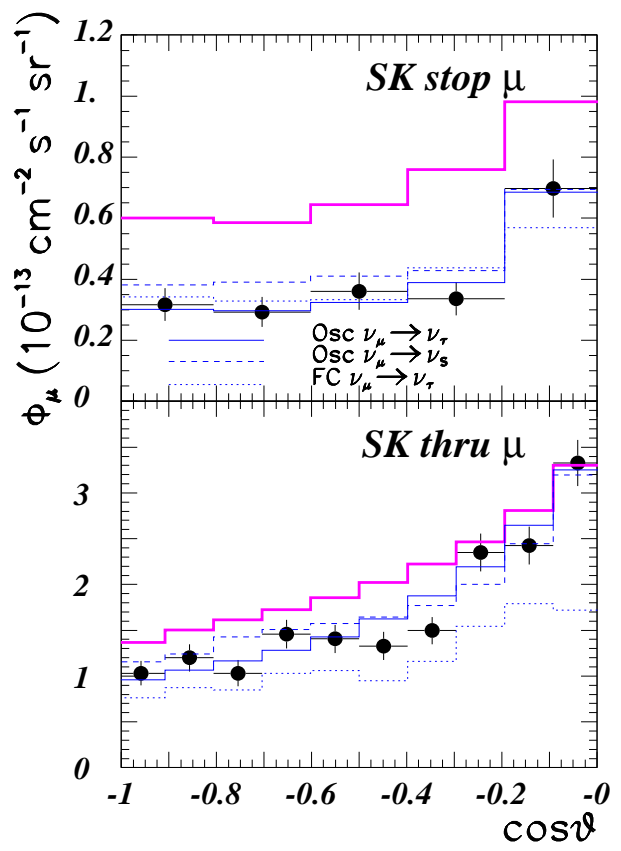

Figure 8: Angular distribution for Super-Kamiokande upgoing muon data together with the SM prediction (no-oscillation) as well as the prediction for the best-fit point to the full data sample for the different conversion mechanisms labelled in the figure.

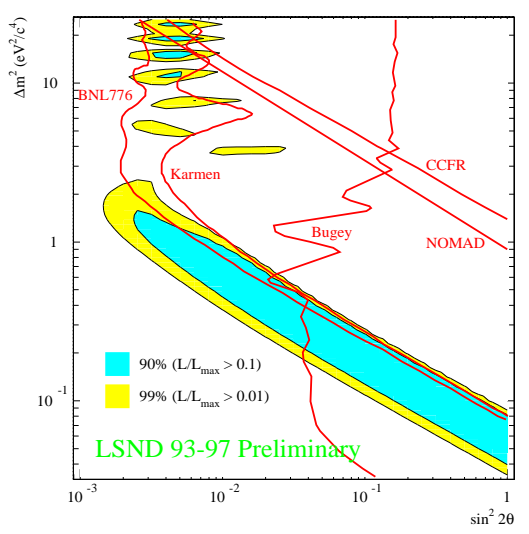

Figure 9: Allowed LSND oscillation parameters versus competing experiments 63 

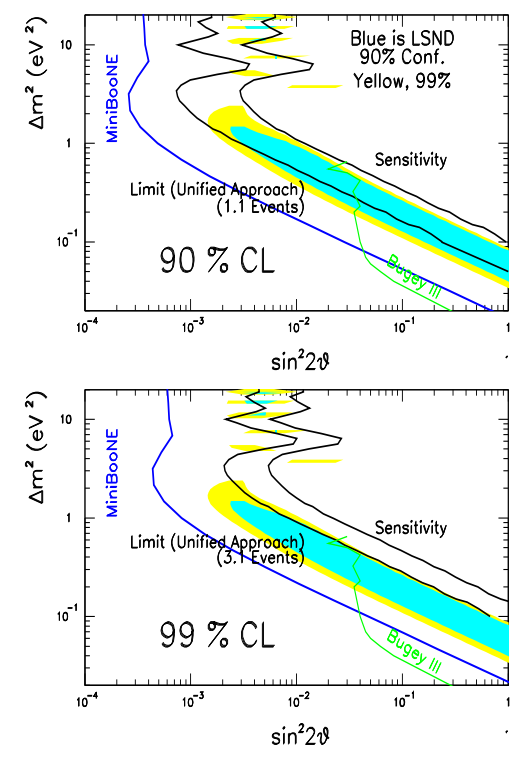

Figure 10: Expected sensitivity of the proposed MiniBooNE experiment 63]

the LSND collaboration. Using $\nu_{\mu}$ from $\pi^{+}$decay in flight, the $\nu_{e}$ appearance is detected via the charged-current reaction $C\left(\nu_{e}, e^{-}\right) X$. Two independent analyses are consistent with the above signature, after taking into account the events expected from the $\nu_{e}$ contamination in the beam and the beam-off background. If interpreted as an oscillation signal, the observed oscillation probability of $2.6 \pm 1.0 \pm 0.5 \times 10^{-3}$, consistent with the evidence for oscillation in the $\bar{\nu}_{\mu} \rightarrow \bar{\nu}_{e}$ channel described above. Fig. 10 compares the LSND region with the expected sensitivity from MiniBooNE, which was recently approved to run at Fermilab [28, 63]. A possible confirmation of the LSND anomaly would be a discovery of far-reaching implications.

\subsection{Dark Matter}

Galaxies as well as the large scale structure in the Universe should arise from the gravitational collapse of fluctuations in the expanding universe. They are sensitive to the nature of the cosmological dark matter. The data on cosmic background temperature anisotropies on large scales performed by the COBE satellite 64 combined with cluster-cluster correlation data e.g. from IRAS 65 can not be reconciled with the simplest COBE-normalized $\Omega_{m}=1$ cold dark matter (CDM) model, since it leads to too much power on small scales. Adding to CDM neutrinos with mass of few $\mathrm{eV}$ (a scale similar to the one indicated by the LSND experiment [27]) corresponding to $\Omega_{\nu} \approx 0.2$, results in an improved fit to data on the nearby galaxy and cluster distribution [66]. The resulting Cold + Hot Dark Matter (CHDM) cosmological model is the most successful $\Omega_{m}=1$ model for structure formation, preferred by inflation. However, other recent data have begun to indicate a lower value for $\Omega_{m}$, thus weakening the cosmological evidence favouring neutrino mass of a few $\mathrm{eV}$ in flat models with cosmological constant $\Omega_{\Lambda}=1-\Omega_{m}$ [66]. Future sky maps of the cosmic microwave background radiation (CMBR) with high precision at the MAP and PLANCK missions should bring more light into 
the nature of the dark matter and the possible rôle of neutrinos [32]. Another possibility is to consider unstable dark matter scenarios [67]. For example, an $\mathrm{MeV}$ range tau neutrino may provide a viable unstable dark matter scenario [68] if the $\nu_{\tau}$ decays before the matter dominance epoch. Its decay products would add energy to the radiation, thereby delaying the time at which the matter and radiation contributions to the energy density of the universe become equal. Such delay would allow one to reduce the density fluctuations on the smaller scales purely within the standard cold dark matter scenario. Upcoming MAP and PLANCK missions may place limits on neutrino stability 69] and rule out such schemes.

\subsection{Pulsar Velocities}

One of the most challenging problems in modern astrophysics is to find a consistent explanation for the high velocity of pulsars. Observations [70] show that these velocities range from zero up to $900 \mathrm{~km} / \mathrm{s}$ with a mean value of $450 \pm 50 \mathrm{~km} / \mathrm{s}$. An attractive possibility is that pulsar motion arises from an asymmetric neutrino emission during the supernova explosion. In fact, neutrinos carry more than $99 \%$ of the new-born proto-neutron star's gravitational binding energy so that even a $1 \%$ asymmetry in the neutrino emission could generate the observed pulsar velocities. This could in principle arise from the interplay between the parity violation present in weak interactions with the strong magnetic fields which are expected during a SN explosion [71, 72]. However, it has recently been noted [73] that no asymmetry in neutrino emission can be generated in thermal equilibrium, even in the presence of parity violation. This suggests that an alternative mechanism is at work. Several neutrino conversion mechanisms in matter have been invoked as a possible engine for powering pulsar motion. They all rely on the polarization [74] of the SN medium induced by the strong magnetic fields $10^{15}$ Gauss present during a SN explosion. This would affect neutrino propagation properties giving rise to an angular dependence of the matter-induced neutrino potentials. This would lead in turn to a deformation of the "neutrino-sphere" for, say, tau neutrinos and thus to an anisotropic neutrino emission. As a consequence, in the presence of non-vanishing $\nu_{\tau}$ mass and mixing the resonance sphere for the $\nu_{e}-\nu_{\tau}$ conversions is distorted. If the resonance surface lies between the $\nu_{\tau}$ and $\nu_{e}$ neutrino spheres, such a distortion would induce a temperature anisotropy in the flux of the escaping tau-neutrinos produced by the conversions, hence a recoil kick of the proto-neutron star. This mechanism was realized in ref. [75] invoking MSW conversions [10]

with $m_{\nu_{\tau}} \gtrsim 100 \mathrm{eV}$ or so, assuming a negligible $\nu_{e}$ mass. This is necessary in order for the resonance surface to be located between the two neutrino-spheres. It should be noted, however, that such requirement is at odds with cosmological bounds on neutrinos masses unless the $\tau$ neutrino is unstable. On the other hand in ref. [76] a realization was proposed in the resonant spin-flavour precession scheme (RSFP) [24]. The magnetic field would not only affect the medium properties, but would also induce the spin-flavour precession through its coupling to the neutrino transition magnetic moment [23.

Perhaps the simplest and probably most elegant suggestion was proposed in ref. [77], where the required pulsar velocities would arise from anisotropic neutrino emission induced by res- 
onant conversions of massless neutrinos (hence no magnetic moment). Raffelt and Janka [78] have subsequently argued that the asymmetric neutrino emission effect was overestimated in [75, 76, 77], since the temperature variation over the deformed neutrino-sphere is not an adequate measure for the anisotropy of the neutrino emission. This would invalidate all neutrino conversion mechanisms, leaving the pulsar velocity problem without any viable solution. However Kusenko and Segré still maintain that sizeable pulsar kicks can arise from neutrino conversions [79]. In any case invoking conversions into sterile neutrinos could be an interesting possibility, since the conversions could take place deeper in the star 80 .

\section{Making Sense of All That}

Physics beyond the Standard Model is required in order to explain solar and atmospheric neutrino data. While neutrino oscillations provide an excellent fit and a powerful way to determine neutrino mass and mixing, there is a plethora of alternative mechanisms, some of which quite attractive, which could play an important rôle in the interpretation of the data. These include flavour changing neutrino interactions both in the solar [15] and atmospheric [16, 81] neutrino problems, Resonant 24, 25] and the Aperiodic Spin-Flavour Precession mechanisms [26] for solar neutrinos, which use the transition magnetic moments of Majorana neutrinos [23], and the possibility of fast neutrino decays [22] which could play a rôle in the atmospheric neutrino problem [82]. Moreover I note that more exotic explanations of the undergound neutrino data based upon violations of equivalence principle, Lorentz invariance and CPT have been proposed [83]. Nevertheless in what follows I will assume the standard neutrino oscillation interpretation of the data.

\subsection{Solar plus Atmospheric}

These data can be accounted for with the three known neutrinos. They fix the two mass splittings $\Delta m_{\odot}^{2} \& \Delta m_{\text {atm }}^{2}$, and two of the three neutrino mixing angles, the third being small on account of the Chooz reactor results [59]. Such scenario can easily be accommodated in seesaw theories of neutrino mass since, in general, the mixing angles involved are not predicted, in particular the maximal mixing indicated by the atmospheric data and possibly also by the solar data can be accomodated by hand. In contrast, it is not easy to reconcile maximal or bimaximal mixing of neutrinos [39] with a predictive quark-lepton unification seesaw scheme that relates lepton and quark mixing angles, since the latter are known to be small. For attempts to reconcile solar and atmospheric data in unified models with specific texture anzatze, see ref. 84, 85.

An alternative way to predict a hierarchical pattern of neutrino mass and mixing, which naturally accomodates the possibility of maximal mixing is to appeal to supersymmetry. In ref. [86] it was shown that the simplest unified extension of the Minimal Supersymmetric Standard Model with bi-linear R-Parity violation provides a predictive scheme for neutrino masses

which can account for the observed atmospheric and solar neutrino anomalies in terms of 
bi-maximal neutrino mixing. The maximality of the atmospheric mixing angle arises dynamically, by minimizing the scalar potential of the theory, while the solar neutrino problem can be accounted for either by large or by small mixing oscillations. The spectrum is naturally hierarchical, since only the tau neutrino picks up mass at the tree level (though this may be itself calculable from renormalization-group evolution from unification down to weak-sacle), by mixing with neutralinos, while the masslessness of the other two neutrinos is lifted only by calculable loop corrections. Despite the smallness of neutrino masses R-parity violation can be observable at present and future high-energy colliders, providing an unambiguous cross-check of the model, and the possibility of probing the neutrino anomalies at accelerators.

Bi-maximal models may also be tested at the upcoming long-baseline experiments or at a possible neutrino factory experiment [87] through the $\mathrm{CP}$ violating phases, which could lead to non-negligible $\mathrm{CP}$ asymmetries in neutrino oscillations [88]. Unfortunately the effects of the $\mathrm{CP}$ violation intrinsic of the Majorana neutrino system [89 is helicity suppressed [9], though a potential test of the CP properties and Majorana nature of neutrinos has been suggested in ref. 90 .

\subsection{Solar and Atmospheric plus Dark Matter}

The story gets more complicated if one wishes to account also for the hot dark matter. The only possibility to fit the solar, atmospheric and HDM scales in a world with just the three known neutrinos is if all of them have nearly the same mass [31], of about $\sim 1.5 \mathrm{eV}$ or so in order to provide the right amount of HDM [66] (all three active neutrinos contribute to HDM). This can be arranged in a unified $S O(10)$ seesaw model where, to first approximation all neutrinos lie at the above HDM mass scale $(\sim 1.5 \mathrm{eV})$, due to a suitable horizontal symmetry, the splittings $\Delta m_{\odot}^{2} \& \Delta m_{\text {atm }}^{2}$ appearing as symmetry breaking effects. An interesting fact is that the ratio $\Delta m_{\odot}^{2} / \Delta m_{\text {atm }}^{2}$ is related to $m_{c}{ }^{2} / m_{t}{ }^{2}$ 91]. There is no room in this case to accommodate the LSND anomaly. To what extent this solution is theoretically natural has been discussed recently in ref. [92]. One finds that the degeneracy is stable in the phenomenologically relevant case where neutrinos have opposite CP parities, leading to a suppression in the neutrino-less doble beta decay rate 93 .

\subsection{Solar \& Atmospheric with Dark Matter \& LSND: Four-Neutrino Models}

An alternative way to include hot dark matter scale is to invoke a fourth light sterile neutrino [29, 30, 31]. As a bonus we can accomodate the LSND hint. The sterile neutrino $\nu_{s}$ must also be light enough in order to participate in the oscillations together with the three active neutrinos. Since it is an $S U(2) \otimes U(1)$ singlet it does not affect the invisible $\mathrm{Z}$ decay width, well-measured at LEP. The theoretical requirements are:

- to understand what keeps the sterile neutrino light, since the $S U(2) \otimes U(1)$ gauge symmetry would allow it to have a large bare mass 


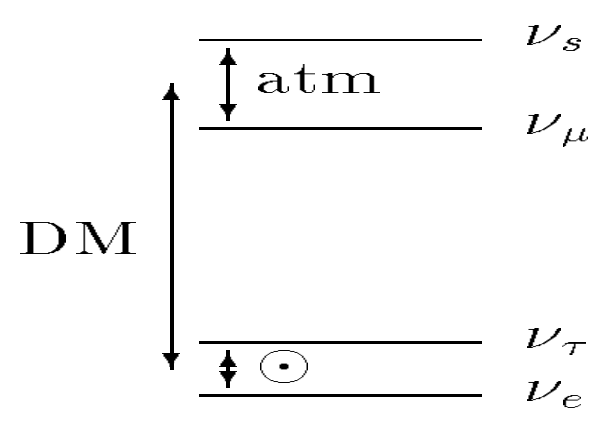

Figure 11: $(e \tau)\left(\begin{array}{ll}\mu & s\end{array}\right)$ scheme: $\nu_{e}-\nu_{\tau}$ conversions explain the solar neutrino data and $\nu_{\mu}$ $\nu_{s}$ oscillations account for the atmospheric deficit, ref. [29].

- to account for the maximal neutrino mixing indicated by the atmospheric data, and possibly by the solar

- to account from first principles for the scales $\Delta m_{\odot}^{2}, \Delta m_{a t m}^{2}$ and $\Delta m_{L S N D / H D M}^{2}$

With this in mind we have formulated the simplest maximally symmetric schemes, denoted as $(e \tau)(\mu s)$ [29] and $(e s)(\mu \tau)$ [30], respectively. One should realize that a given scheme (mainly the structure of the leptonic charged current) may be realized in more than one theoretical model. For example, an alternative to the model in [30] was suggested in ref. [31]. Higher dimensional theories contain light sterile neutrinos which can arise from the bulk sector and reproduce the basic features of these models [94]. For a recent discussion of the experimental constraints on four-neutrino mixing see ref. [95]. For alternative theoretical and phenomenological scenarios see ref. 96, 97.

Although many of the phenomenological features arise also in other models, here I concentrate the discussion mainly on the theories developed in ref. [29, 30]. These are characterized by a very symmetric mass spectrum in which there are two ultra-light neutrinos at the solar neutrino scale and two maximally mixed almost degenerate eV-mass neutrinos (the LSND/HDM scale [98]), split by the atmospheric neutrino scale [29, 30]. Before the global U(1) lepton symmetry breaks the heaviest neutrinos are exactly degenerate, while the other two are massless [99]. After the U(1) breaks down the heavier neutrinos split and the lighter ones get mass. The scale $\Delta m_{L S N D / H D M}^{2}$ is generated radiatively at one-loop due to the additional Higgs bosons, while the splittings $\Delta m_{\text {atm }}^{2}$ and $\Delta m^{2} \odot$ are two-loop effects. The models are based only on weak-scale physics: no large mass scale is introduced. They explain the lightness of the sterile neutrino 1 , the large mixing required by the atmospheric neutrino data, as well as the generation of the mass splittings responsible for solar and atmospheric neutrino conversions as natural consequences of the underlying lepton-number symmetry and its breaking. They are minimal in the sense that they add a single $S U(2) \otimes U(1)$ singlet lepton to the SM. The models differ according to whether the $\nu_{s}$ lies at the dark matter scale or at the solar neutrino scale. In the $(e \tau)(\mu s)$ scheme the $\nu_{s}$ lies at the LSND/HDM scale, as illustrated in Fig. (11) while in

\footnotetext{
${ }^{1}$ In higher dimensional theries such sterile neutrinos may arise from bulk matter and be light without need for a protecting symmetry, see ref. 94 .
} 


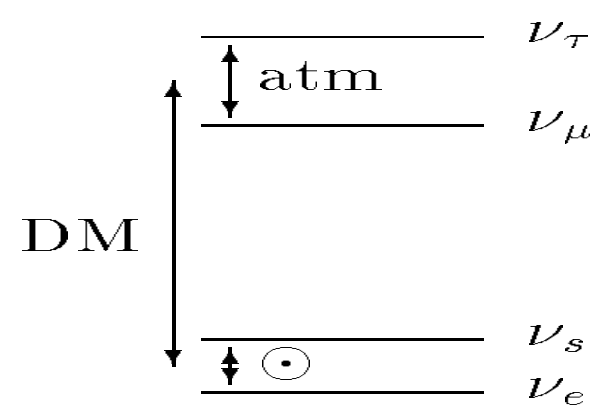

Figure 12: $(e s)(\mu \tau)$ scheme: $\nu_{e}-\nu_{s}$ conversions explain the solar neutrino data and $\nu_{\mu}$ $\nu_{\tau}$ oscillations account for the atmospheric deficit, ref. [30.

the alternative $(e s)(\mu \tau)$ model, $\nu_{s}$ is at the solar neutrino scale as shown in Fig. (12) [30]. In the $(e \tau)(\mu s)$ case the atmospheric neutrino puzzle is explained by $\nu_{\mu}$ to $\nu_{s}$ oscillations, while in $(e s)(\mu \tau)$ it is due to $\nu_{\mu}$ to $\nu_{\tau}$ oscillations. Correspondingly, the deficit of solar neutrinos is explained in the first case by $\nu_{e}$ to $\nu_{\tau}$ conversions, while in the second the relevant channel is $\nu_{e}$ to $\nu_{s}$. In both models [29, 30] one predicts close-to-maximal mixing in the atmospheric neutrino sector, a feature which emerges as the global best-fit points in the analyses discussed above.

The presence of additional weakly interacting light particles, such as our light sterile neutrino, is constrained by BBN since the $\nu_{s}$ would enter into equilibrium with the active neutrinos in the early Universe (and therefore would contribute to $N_{\nu}^{\max }$ ) via neutrino oscillations [100], unless $\Delta m^{2} \sin ^{4} 2 \theta \lesssim 3 \times 10^{-6} \mathrm{eV}^{2}$. Here $\Delta m^{2}$ denotes a typical mass-square difference of the active and sterile species and $\theta$ is the vacuum mixing angle. However, systematic uncertainties in the BBN bounds still caution us not to take them too literally. For example, it has been argued that present observations of primordial Helium and deuterium abundances may allow up to $N_{\nu}=4.5$ neutrino species if the baryon to photon ratio is small [101. Adopting this as a limit, clearly both models described above are consistent. Should the BBN constraints get tighter 102 e.g. $N_{\nu}^{\max }<3.5$ they could rule out the $(e \tau)(\mu s)$ model, and leave out only the competing scheme as a viable alternative. However the possible rôle of a primordial lepton asymmetry might invalidate this conclusion, for recent work on this see ref. 103.

It is well-known that the neutral-to-charged current ratios are important observables in neutrino oscillation phenomenology, especially sensitive to the existence of singlet neutrinos, light or heavy [89]. On this basis the two models above would be distinguishable at future neutral-current-sensitive solar and atmospheric neutrino experiments. For example they may be tested in the SNO experiment [104] once they measure the solar neutrino flux $\left(\Phi_{\nu}^{N C}\right)$ in their neutral current data and compare it with the corresponding $C \mathrm{C}$ value $\left(\Phi_{\nu}^{C C}\right)$. If the solar neutrinos convert to active neutrinos, as in the $(e \tau)(\mu s)$ model, then one expects $\Phi_{\nu}^{C C} / \Phi_{\nu}^{N C}$ around 0.5 , whereas in the $(e s)(\mu \tau)$ scheme $\left(\nu_{e}\right.$ conversion to $\left.\nu_{s}\right)$, the above ratio would be nearly $\simeq 1$. Looking at pion production via the neutral current reaction $\nu_{\tau}+N \rightarrow \nu_{\tau}+\pi^{0}+N$ in the atmospheric data might also help in distinguishing between these two possibilities [105], since this reaction is absent in the case of sterile neutrinos, but would exist in the $(e s)(\mu \tau)$ 
scheme.

If light sterile neutrinos indeed exist one can show that they might contribute to a cosmic hot dark matter component and to an increased radiation content at the epoch of matter-radiation equality. These effects leave their imprint in sky maps of the cosmic microwave background radiation $(\mathrm{CMBR})$ and may thus be detectable with the very high precision measurements expected at the upcoming MAP and PLANCK missions as noted in ref. 32].

\subsection{Heavy Tau Neutrino}

Finally, the door is not closed to heavy neutrinos. Indeed, an alternative to the inclusion of hot dark matter is to simulate its effects through the late decay of an $\mathrm{MeV}$ tau neutrino [68], in the presence of a light sterile neutrino. Indeed such a model was presented 106 where an unstable $\mathrm{MeV}$ Majorana tau neutrino naturally reconciles the cosmological observations of large and small-scale density fluctuations with the cold dark matter picture. The model assumes the spontaneous violation of a global lepton number symmetry at the weak scale. The breaking of this symmetry generates the cosmologically required decay of the $\nu_{\tau}$ with lifetime $\tau_{\nu_{\tau}} \sim 10^{2}-10^{4} \mathrm{sec}$, as well as the masses and oscillations of the three light neutrinos $\nu_{e}$, $\nu_{\mu}$ and $\nu_{s}$ which may account for the present solar and atmospheric data, though a dedicated three-neutrino fit in which one of the neutrinos is sterile would be desirable.

\section{In conclusion}

The angle-dependent atmospheric neutrino deficit provides, together with the solar neutrino data, a strong evidence for physics beyond the Standard Model. Small neutrino masses provide the simplest, but not unique, explanation of the data. Allowing for alternative explanations of the underground experiments involving non-standard neutrinos opens new possibilities involving either massless or even very heavy cosmologically unstable neutrinos, which naturally arise in many models. From this point of view, it is still too early to infer with great certainty neutrino masses and angles from underground experiments alone. Keeping within the framework of the standard neutrino oscillation interpretation of the data, one has an interesting possibility of bi-maximal neutrino mixing and of testing the neutrino anomalies not only at the upcoming long-baseline or neutrino factory experiments, but also at high-energy accelerators. On the other hand if the LSND result stands the test of time, this would be a strong indication for the existence of a light sterile neutrino. The two most attractive ways to reconcile underground observations with LSND invoke either $\nu_{e}-\nu_{\tau}$ conversions to explain the solar data, with $\nu_{\mu}-$ $\nu_{s}$ oscillations accounting for the atmospheric deficit, or the opposite. At the moment the latter is favored by the atmospheric data. These two basic schemes have distinct implications at future neutral-current-sensitive solar \& atmospheric neutrino experiments, such as SNO and Super-Kamiokande. To end up on a phylosophical mood I would say that it is important to search for manifestations of massive and/or non-standard neutrinos at the laboratory in an unbiased way. Though most of the recent excitement now comes from underground experiments, 
one should bear in mind that models of neutrino mass may lead to a plethora of new signatures which may be accessible also at high-energy accelerators, thus illustrating the complementarity between the two approaches.

I am grateful to the Organizers for the kind hospitality and to all my collaborators, especially Concha Gonzalez-Garcia and her student Carlos Peña for the re-analysis of solar neutrino data. This work was supported by DGICYT grant PB95-1077 and by the TMR contract ERBFMRXCT96-0090.

\section{References}

[1] B.T. Cleveland et al., Astrophys. J. 496, 505 (1998).

[2] GAlleX Collaboration (W. Hampel et al.), Phys. Lett. B447, 127 (1999); T. Kirsten, Talk at the Sixth international workshop on topics in astroparticle and underground physics September, TAUP99, Paris, September 1999; SAGE Collaboration (J.N. Abdurashitov et al.), Phys. Rev. C 59, 2246 (1999); SAGE Collaboration (J.N. Abdurashitov et al.), astro-ph/9907113.

[3] Y. Fukuda et al. [Super-Kamiokande Collaboration], Phys. Rev. Lett. 82, 1810 (1999) hepex/9812009; Super-Kamiokande Collaboration, Y. Fukuda et al., Phys. Rev. Lett. 82, 2430 (1999).

[4] Y. Suzuki, talk a the "XIX International Symposium on Lepton and Photon Interactions at High Energies", Stanford University, August 9-14, 1999.

[5] NUSEX Collaboration, M. Aglietta et al., Europhys. Lett. 8, 611 (1989); Fréjus Collaboration, Ch. Berger et al., Phys. Lett. B227, 489 (1989); IMB Collaboration, D. Casper et al., Phys. Rev. Lett. 66, 2561 (1991); R. Becker-Szendy et al., Phys. Rev. D46, 3720 (1992); Kamiokande Collaboration, H. S. Hirata et al., Phys. Lett. B205, 416 (1988) and Phys. Lett. B280, 146 (1992); Kamiokande Collaboration, Y. Fukuda et al., Phys. Lett. B335, 237 (1994); Soudan Collaboration, W. W. M Allison et al., Phys. Lett. B391, 491 (1997)

[6] Y. Fukuda et al. [Super-Kamiokande Collaboration], "Evidence for oscillation of atmospheric neutrinos," Phys. Rev. Lett. 81, 1562 (1998) hep-ex/9807003; Y. Fukuda et al., Phys. Rev. Lett. 82, 2644 (1998), hep-ex/9812014 and Y. Fukuda et al. hep-ex/9908049. The most recent 52 kton-yr data were presented in the invited talk by A. Mann at the XIX Lepton Photon Symposium at Stanford University, August 1999.

[7] For a review see J. W. F. Valle, Gauge Theories and the Physics of Neutrino Mass, Prog. Part. Nucl. Phys. 26 (1991) 91-171

[8] J. Schechter and J.W. Valle, Phys. Rev. D25, 2951 (1982); for reviews see A. Morales, "Review on double beta decay experiments and comparison with theory," hep-ph/9809540

[9] J. Schechter and J.W. Valle, Phys. Rev. D23 (1981) 1666.

[10] A.Y. Smirnov and S.P. Mikheev, "Neutrino Oscillations In Matter With Varying Density," In *Tignes 1986, Proceedings, '86 massive neutrinos* 355-372; L. Wolfenstein, Phys. Rev. D20 (1979) 2634.

[11] M.C. Gonzalez-Garcia, P.C. de Holanda, C. Pena-Garay, and J. W. F. Valle, Status of the MSW Solutions of the Solar Neutrino Problem, hep-ph/9906469, to be published in Nucl. Phys. B

[12] J.W. Valle, Phys. Lett. 199B (1987) 432.

[13] M.M. Guzzo and S.T. Petcov, Phys. Lett. B271 (1991) 172.

[14] H. Nunokawa, Y.Z. Qian, A. Rossi and J.W. Valle, Phys. Rev. D54 (1996) 4356 hep-ph/9605301.

[15] P.I. Krastev and J.N. Bahcall, "FCNC solutions to the solar neutrino problem," hep-ph/9703267.

[16] M.C. Gonzalez-Garcia et al., Phys. Rev. Lett. 82 (1999) 3202, hep-ph/9809531. 
[17] D. Wyler, L. Wolfenstein, Nucl. Phys. B218 (1983) 205

[18] R. Mohapatra, J. W. F. Valle, Phys. Rev. D34 (1986) 1642; J. W. F. Valle, Nucl. Phys. B (Proc. Suppl.) B11 (1989) 118-177

[19] J. Bernabeu, A. Santamaria, J. Vidal, A. Mendez, J. W. F. Valle, Phys. Lett. B187 (1987) 303; J. G. Korner, A. Pilaftsis, K. Schilcher, Phys. Lett. B300 (1993) 381

[20] M. C. Gonzalez-Garcia, J. W. F. Valle, Mod. Phys. Lett. A7 (1992) 477; erratum Mod. Phys. Lett. A9 (1994) 2569; A. Ilakovac, A. Pilaftsis, Nucl. Phys. B437 (1995) 491; A. Pilaftsis, Mod. Phys. Lett. A9 (1994) 3595

[21] L.J. Hall, V.A. Kostelecky and S. Raby, Nucl. Phys. B267, 415 (1986).

[22] J. W. F. Valle, Phys. Lett. B131 (1983) 87; G. Gelmini, J. W. F. Valle, Phys. Lett. B142 (1984) 181; J. W. F. Valle, Phys. Lett. B159 (1985) 49; A. Joshipura, S. Rindani, Phys. Rev. D46 (1992) 3000

[23] J. Schechter, J. W. F. Valle, Phys. Rev. D24 1883, (1981), Err. ibid.D25 283, (1982).

[24] E.Kh. Akhmedov, Phys. Lett. B213 (1988) 64-68; C. S. Lim and W. Marciano, Phys. Rev. D37 (1988) 1368

[25] E.Kh. Akhmedov, The neutrino magnetic moment and time variations of the solar neutrino flux, hep-ph/9705451

[26] V.B. Semikoz, A.A. Bykov, V.Y. Popov, A.I. Rez and D.D. Sokoloff, hep-ph/9808274.

[27] C. Athanassopoulos, [LSND Collaboration], Phys. Rev. Lett. 75 (1995) 2650; Phys. Rev. Lett. 77 (1996) 3082 ; C. Athanassopoulos et al, Phys. Rev. Lett. 81, 1774 (1998)

[28] W.C. Louis [LSND Collaboration], Prog. Part. Nucl. Phys. 40, 151 (1998).

[29] J. T. Peltoniemi, D. Tommasini, and J W F Valle, Phys. Lett. B298 (1993) 383

[30] J. T. Peltoniemi, and J W F Valle, Nucl. Phys. B406 (1993) 409

[31] D.O. Caldwell and R.N. Mohapatra, Phys. Rev. D48 (1993) 3259

[32] S. Hannestad and G. Raffelt, Phys. Rev. D59, 043001 (1999) astro-ph/9805223.

[33] J. N. Bahcall, astro-ph/9808162

[34] J. N. Bahcall, S. Basu and M. H. Pinsonneault, Phys. Lett. B 433 (1998) 1.

[35] (GONG) J. Christensen-Dalsgaard et al., GONG Collaboration, Science 272 (1996) 1286; (BP95) J. N. Bahcall and M. H. Pinsonneault, Rev. Mod. Phys. 67 (1995) 781; (KS94) A. Kovetz and G. Shaviv, Astrophys. J. 426 (1994) 787; (CDF94) V. Castellani, S. Degl'Innocenti, G. Fiorentini, L.M. Lissia and B. Ricci, Phys. Lett. B 324 (1994) 425; (JCD94) J. Christensen-Dalsgaard, Europhys. News 25 (1994) 71; (SSD94) X. Shi, D.N. Schramm and D.S.P. Dearborn, Phys. Rev. D 50 (1994) 2414; (DS96) A. Dar and G. Shaviv, Astrophys. J. 468 (1996) 933; (CDF93) V. Castellani, S. Degl'Innocenti and G. Fiorentini, Astron. Astrophys. 271 (1993) 601; (TCL93) S. Turck-Chièze and I. Lopes, Astrophys. J. 408 (1993) 347; (BPML93) G. Berthomieu, J. Provost, P. Morel and Y. Lebreton, Astron. Astrophys. 268 (1993) 775; (BP92) J.N. Bahcall and M.H. Pinsonneault, Rev. Mod. Phys. 64 (1992) 885; (SBF90) I.-J. Sackman, A.I. Boothroyd and W.A. Fowler, Astrophys. J. 360 (1990) 727; (BU88) J.N. Bahcall and R.K. Ulrich, Rev. Mod. Phys. 60 (1988) 297; (RVCD96) O. Richard, S. Vauclair, C. Charbonnel and W.A. Dziembowski, Astron. Astrophys. 312 (1996) 1000; (CDR97) F. Ciacio, S. Degl'Innocenti and B. Ricci, Astron. Astrophys. Suppl. Ser. 123 (1997) 449.

[36] J. N. Bahcall, Phys. Lett. B338 (1994) 276; V. Castellani, et al Phys. Lett. B324 (1994) 245; N. Hata, S. Bludman, and P. Langacker, Phys. Rev. D49 (1994) 3622; V. Berezinsky, Comm. on Nucl. and Part. Phys. 21 (1994) 249

[37] J.N. Bahcall, M.H. Pinsonneault, S. Basu and J. Christensen-Dalsgaard, Phys. Rev. Lett. 78 (1997) 171. 
[38] V. Barger, K. Whisnant and R.J. Phillips, Phys. Rev. D24, 538 (1981); S.L. Glashow and L.M. Krauss, Phys. Lett. 190B, 199 (1987); S.L. Glashow, P.J. Kernan and L.M. Krauss, Phys. Lett. B445, 412 (1999)

[39] V. Barger, S. Pakvasa, T.J. Weiler and K. Whisnant, Phys. Lett. B437, 107 (1998), hepph/9806387; E. Torrente-Lujan, Phys. Lett. B389 (1996) 557; S. Davidson and S.F. King, Phys. Lett. B445, 191 (1998).

[40] J.N. Bahcall and P.I. Krastev, Phys. Lett. B436, 243 (1998); R. Escribano, J.M. Frere, A. Gevaert and D. Monderen, Phys. Lett. B444, 397 (1998).

[41] G. Fiorentini, V. Berezinsky, S. Degl'Innocenti and B. Ricci, "Bounds on hep neutrinos," Phys. Lett. B444, 387 (1998), astro-ph/9810083.

[42] P.C. de Holanda, C. Pena-Garay, M.C. Gonzalez-Garcia and J. W. F. Valle, "Seasonal dependence in the solar neutrino flux," Phys. Rev. D60 (1999) 093010, hep-ph/9903473; see also J.N. Bahcall, P.I. Krastev and A.Y. Smirnov, hep-ph/9905220.

[43] A.B. Balantekin, J.M. Fetter and F.N. Loreti, Phys. Rev. D54 (1996) 3941-3951; F. N. Loreti and A. B. Balantekin, Phys. Rev. D50 (1994) 4762; F. N. Loreti et al., Phys. Rev. D52 (1996) 6664.

[44] H. Nunokawa, A. Rossi, V. Semikoz, J. W. F. Valle, Nucl. Phys. B472 (1996) 495-517 [see also talk at Neutrino 96, hep-ph/9610526

[45] P. Bamert, C.P. Burgess and D. Michaud, Nucl. Phys. B513, 319 (1998); C.P. Burgess, hepph/9711425; C.P. Burgess and D. Michaud, Annals Phys. 256, 1 (1997) and hep-ph/9611368.

[46] C. Arpesella at al., Proposal of the Borexino experiment (1991).

[47] V. Barger, K. Whisnant, hep-ph/9903262; S. Goswami, D. Majumdar and A. Raychaudhuri, hep$\mathrm{ph} / 9909453$.

[48] S.P. Mikheyev, A.Yu. Smirnov Phys.Lett. B429 (1998) 343-348; B. Faid, G. L. Fogli, E. Lisi, D. Montanino, hep-ph/9805293

[49] Y. Fukuda et al., Phys. Lett. B433, 9 (1998); Phys. Lett. B436, 33 (1998)

[50] M. Nakahata, talk at Sixth International Workshop on Topics in Astroparticle and Underground Physics, TAUP99, Paris September 1999.

[51] K. Daum et al. Z. Phys. C66, 417 (1995).

[52] M. Aglietta et al., Europhys. Lett. 8, 611 (1989).

[53] H. S. Hirata et al., Phys. Lett. B280, 146 (1992); Y. Fukuda et al., ibid B335, 237 (1994).

[54] R. Becker-Szendy et al., Phys. Rev. D46, 3720 (1992).

[55] W. W. M. Allison et al., Phys. Lett. B449, 137 (1999).

[56] T. K. Gaisser, F. Halzen and T. Stanev, Phys. Rep. 258, 174 (1995)

[57] T. K. Gaisser and T. Stanev, Phys. Rev. D57 1977 (1998); G. Barr, T. K. Gaisser and T. Stanev, Phys. Rev. D 39 (1989) 3532 and Phys. Rev. D38, 85; V. Agrawal et al., Phys. Rev. D53, 1314 (1996); L. V. Volkova, Sov. J. Nucl. Phys. 31, 784 (1980); M. Honda, T. Kajita, S. Midorikawa and K. Kasahara, Phys. Rev. D52, 4985 (1995).

[58] M. C. Gonzalez-Garcia, H. Nunokawa, O. L. G. Peres, T. Stanev, J. W. F. Valle, Phys. Rev. D58 (1998) 033004 [hep-ph 9801368]; for the 535 days data sample update, and the comparison of active and sterile channels see M.C. Gonzalez-Garcia, H. Nunokawa, O.L. Peres and J. W. F. Valle, Nucl. Phys. B543, 3 (1998), hep-ph/9807305.

[59] M. Appollonio et al. Phys. Lett. B420 397(1998), hep-ex/9711002

[60] M. C. Gonzalez-Garcia, talk at International Workshop on Particles in Astrophysics and Cosmology: From Theory to Observation, Valencia, Spain, May 3-8, 1999, To be published in Nucl. Phys. B (Proc. Suppl.), Ed. V. Berezinsky, G. Raffelt and J. W. F. Valle. 
[61] R. Foot, R. R. Volkas, O. Yasuda, TMUP-HEL-9801; O. Yasuda, Phys. Rev. D58, 091301 (1998); G. L. Fogli, E. Lisi, A. Marrone, G. Scioscia, Phys. Rev. D59, 033001 (1999) hep-ph/9808205; E.Kh. Akhmedov, A. Dighe, P. Lipari and A.Yu. Smirnov, hep-ph/9808270

[62] P. Lipari, M. Lusignoli, Phys. Rev. D60, 013003 (1999) and Phys. Rev. D58, 073005 (1998).

[63] W. Louis, http://www.neutrino.lanl.gov/LSND/

[64] G. F. Smoot et al., Astrophys. J. 396 (1992) L1-L5; E.L. Wright et al., Astrophys. J. 396 (1992) L13

[65] R. Rowan-Robinson, in Cosmological Dark Matter, (World Scientific, 1994), ed. A. Perez, and J. W. F. Valle, p. 7-18, ISBN 981-02-1879-6

[66] J.R. Primack and M.A. Gross, astro-ph/9810204; E. Gawiser and J. Silk, astro-ph/9806197; Science, 280, 1405 (1998), and references therein.

[67] G. Gelmini, D.N. Schramm and J. W. F. Valle, Phys. Lett. 146B, 311 (1984).

[68] J. Bond and G. Efstathiou, Phys. Lett. B265 (1991) 245; M. Davis et al., Nature 356 (1992) 489; S. Dodelson, G. Gyuk and M. Turner, Phys. Rev. Lett. 72 (1994) 3754; H. Kikuchi and E. Ma, Phys. Rev. D51 (1995) 296; H. B. Kim and J. E. Kim, Nucl. Phys. B433 (1995) 421; M. White, G. Gelmini and J. Silk, Phys. Rev. D51 (1995) 2669; A. Masiero, D. Montanino and M. Peloso, hep-ph/9902380.

[69] S. Hannestad, "Probing neutrino decays with the cosmic microwave background," Phys. Rev. D59, 125020 (1999) astro-ph/9903475.

[70] A.G. Lyne and D.R. Lorimer, Nature 369 (1994) 127.

[71] N.N. Chugai, Pis'ma Astron. Zh.10, 87 (1984).

[72] A. Vilenkin, Astrophys. J. 451 (1995) 700; Dong Lai, Y.-Z. Qian, astro-ph/9712043

[73] A. Kusenko, G. Segre and A. Vilenkin, "Neutrino transport: No asymmetry in equilibrium," Phys. Lett. B437, 359 (1998) astro-ph/9806205.

[74] H. Nunokawa, V.B. Semikoz, A.Yu. Smirnov and J. W. F. Valle, Nucl. Phys. B501 (1997) 17

[75] A. Kusenko, G. Segrè, Phys. Rev. Lett. 77 (1996) 4872 \& Phys. Rev. Lett. 79 (1997) 2751; Y.Z. Qian, Phys. Rev. Lett. 79 (1997) 2750

[76] E.Kh. Akhmedov, A. Lanza and D.W. Sciama, Phys. Rev. D56 (1997) 6117

[77] D. Grasso, H. Nunokawa and J. W. F. Valle, Phys. Rev. Lett. 81, 2412 (1998), astro-ph/9803002.

[78] H.T. Janka and G.G. Raffelt, Phys. Rev. D59, 023005 (1999) astro-ph/9808099.

[79] A. Kusenko and G. Segre, Phys. Rev. D59 (1999) 061302, astro-ph/9811144.

[80] D. Grasso, H. Nunokawa, A . Rossi, A. Yu. Smirnov, J. W. F. Valle, in preparation

[81] N. Fornengo, M. C. Gonzalez-Garcia, J. W. F. Valle, hep-ph/9906539.

[82] V. Barger et al, hep-ph/9907421 and Phys. Rev. Lett. 82, 2640 (1999)

[83] M. Gasperini, Phys. Rev. D38, 2635 (1988); J. Pantaleone, A. Halprin, C.N. Leung, Phys. Rev. D47, 4199 (1993); A. Halprin, C.N. Leung, J. Pantaleone Phys. Rev. D53, 5365 (1996). S. Coleman, S. L. Glashow, Phys. Lett. B405, 249 (1997); S. L. Glashow, A. Halprin, P.I. Krastev, C.N. Leung, J. Pantaleone, Phys. Rev. D56, 2433 (1997). S. Coleman, S. L. Glashow, Phys. Rev. D59, 116008 (1999). A.M. Gago, H. Nunokawa and R. Zukanovich Funchal, hep-ph/9909250.

[84] S. Lola and J.D. Vergados, Prog. Part. Nucl. Phys. 40, 71 (1998); G. Altarelli and F. Feruglio, Phys. Lett. B439, 112 (1998) hep-ph/9807353.

[85] G. Altarelli and F. Feruglio, Phys. Lett. B451 (1999) 388 hep-ph/9812475; S. Lola and G.G. Ross, hep-ph/9902283; R. Barbieri, L.J. Hall and A. Strumia, Phys. Lett. B445, 407 (1999), hepph/9808333; M.E. Gomez, G.K. Leontaris, S. Lola and J.D. Vergados, Phys. Rev. D59, 116009 (1999), hep-ph/9810291; G.K. Leontaris, S. Lola, C. Scheich and J.D. Vergados, Phys. Rev. D53, $6381(1996)$ 
[86] J.C. Romao, M.A. Diaz, M. Hirsch, W. Porod and J.W. Valle, hep-ph/9907499.

[87] C. Quigg, Introduction to NuFact '99, the ICFA/ECFA Workshop on Neutrino Factories Based on Muon Storage Rings, hep-ph/9908357.

[88] J. Sato, hep-ph/9910442; O. Yasuda, hep-ph/9910428; H. Minakata and H. Nunokawa, Phys. Rev. D57 (1998) 4403 hep-ph/9705208; A. Romanino, hep-ph/9909425.

[89] J. Schechter and J. W. F. Valle, Phys. Rev. D22 (1980) 2227

[90] S. Pastor, J. Segura, V.B. Semikoz and J.W. Valle, hep-ph/9905405, to be published in Nucl. Phys. $\mathrm{B}$

[91] A. Ioannissyan, J. W. F. Valle, Phys. Lett. B332 (1994) 93-99; B. Bamert, C.P. Burgess, Phys. Lett. B329 (1994) 289; D. Caldwell and R. N. Mohapatra, Phys. Rev. D50 (1994) 3477; D. G. Lee and R. N. Mohapatra, Phys. Lett. B329 (1994) 463; A. S. Joshipura, Zeit. fur Physik C64 (1994) 31

[92] J.A. Casas, J.R. Espinosa, A. Ibarra and I. Navarro, hep-ph/9905381; J. Ellis and S. Lola, hepph/9904279; N. Haba, Y. Matsui, N. Okamura and M. Sugiura, hep-ph/9908429.

[93] E. Ma, hep-ph/9907503.

[94] R. N. Mohapatra, A. Perez-Lorenzana, hep-ph/9910474; A. Ioannissyan, J. W. F. Valle, in preparation

[95] C. Giunti, hep-ph/9909465.

[96] Q. Y. Liu, A. Yu. Smirnov, Nucl. Phys. B524 (1998) 505-523; V. Barger, K. Whisnant and T. Weiler, Phys.Lett. B427 (1998) 97-104; S. Gibbons, R. N. Mohapatra, S. Nandi and A. Raichoudhuri, Phys. Lett. B430 (1998) 296-302; Nucl.Phys. B524 (1998) 505-523; S. Bilenky, C. Giunti and W. Grimus, Eur. Phys. J. C 1, 247 (1998); S. Goswami, Phys. Rev. D 55, 2931 (1997); N. Okada and O. Yasuda, Int. J. Mod. Phys. A12 (1997) 3669-3694

[97] E. J. Chun, A. Joshipura and A. Smirnov, in Elementary Particle Physics: Present and Future (World Scientific, 1996), ISBN 981-02-2554-7; P. Langacker, Phys. Rev. D58, 093017 (1998); M. Bando and K. Yoshioka, Prog. Theor. Phys. 100, 1239 (1998)

[98] J. R. Primack, et al. Phys. Rev. Lett. 74 (1995) 2160

[99] J. Schechter and J. W. F. Valle, Phys. Rev. D21 (1980) 309

[100] R. Barbieri and A. Dolgov, Phys. Lett. B 237, 440 (1990); K. Enquist, K. Kainulainen and J. Maalampi, Phys. Lett. B 249, 531 (1992); D. P. Kirilova and M. Chizov, hep-ph/9707282.

[101] S. Sarkar, Rep. Prog. Phys. 59, 1493 (1996); P. J. Kernan and S. Sarkar, Phys. Rev. D 54 (1996) R3681

[102] G. Fiorentini, E. Lisi, S. Sarkar and F.L. Villante, "Quantifying uncertainties in primordial nucleosynthesis without Monte Carlo simulations," Phys. Rev. D58, 063506 (1998); E. Lisi, S. Sarkar and F.L. Villante, Phys. Rev. D59, 123520 (1999)

[103] R. Foot, R.R. Volkas, Phys. Rev. D55 (1997) 5147-5176; A.D. Dolgov, S.H. Hansen, S. Pastor and D.V. Semikoz, hep-ph/9910444.

[104] SNO collaboration, E. Norman et al. Proc. of The Fermilab Conference: DPF 92 ed. C. Albright, P. H. Kasper, R. Raja and J. Yoh (World Scientific), p. 1450.

[105] A. Smirnov and F. Vissani, Phys.Lett. B432 (1998) 376; J. G. Learned, S. Pakvasa and J. Stone, hep-ph/9805343; H. Murayama and L. Hall, hep-ph/9806218

[106] A. Joshipura, J. W. F. Valle, Nucl. Phys. B440 (1995) 647. 